\title{
Benchmarks
}

\section{Induction of Cell Death by Hydrophobic Hydrocarbons}

BioTechniques 26:864-868 (May 1999)

We present a novel and simple method for inducing cell death. Cells placed in contact with paraffin or other hydrophobic-hydrocarbon substances die. Cell death can be the result of apoptosis, which is also known as programmed cell death (PCD). In apoptosis, cells shrink and undergo both disintegration of the nucleoli and nuclear fragmentation (2). In necrosis, cells swell and disintegrate. Apoptosis is a critical homeostatic mechanism in the body to maintain the necessary proportion of cell proliferation and cell death (10). It is a form of cell suicide. The apoptotic pathway was first elucidated in the Caenorhabditis elegans nematode. A number of critical genes (5) and enzymes, notably the caspases (3), are operative and important to the apoptotic process. PCD can be induced by a variety of physical and chemical means (1). We offer yet another method that is both simple and nontoxic. Paraffin, long used in association with dead cells as a tissue-embedding agent, ironically can be used as a method for inducing cell death.

Our experimental protocol consisted of four phases. First, we prepared 1.5-2.0 cc of liquid paraffin (Tissue Prep $^{\circledR}$ 2; Fisher Scientific, Pittsburgh, PA, USA) dispensed into polystyrene 6-well tissue culture plates (Falcon ${ }^{\circledR}$ Model 3046; Becton Dickinson Labware, Bedford, MA, USA). The paraffin, when cool, formed uniform solid smooth surfaces at the bottom of the wells. Cell controls consisted of plain wells not coated with paraffin and treated identically. The following four cell lines were used: (i) human fetal lung (MRC-5, Catalog No. CCL-171; ATCC, Rockville, MD, USA), (ii) human lung carcinoma (A549, Catalog No. CCL-185; ATCC), (iii) human rhabdomyosarcoma (RD, Catalog No. CCL-136; ATCC) and (iv) human breast carcinoma (MCF 7, Catalog No. HTB-22; ATCC). Confluent monolayers from $75-\mathrm{cm}^{2}$ tissue culture flasks

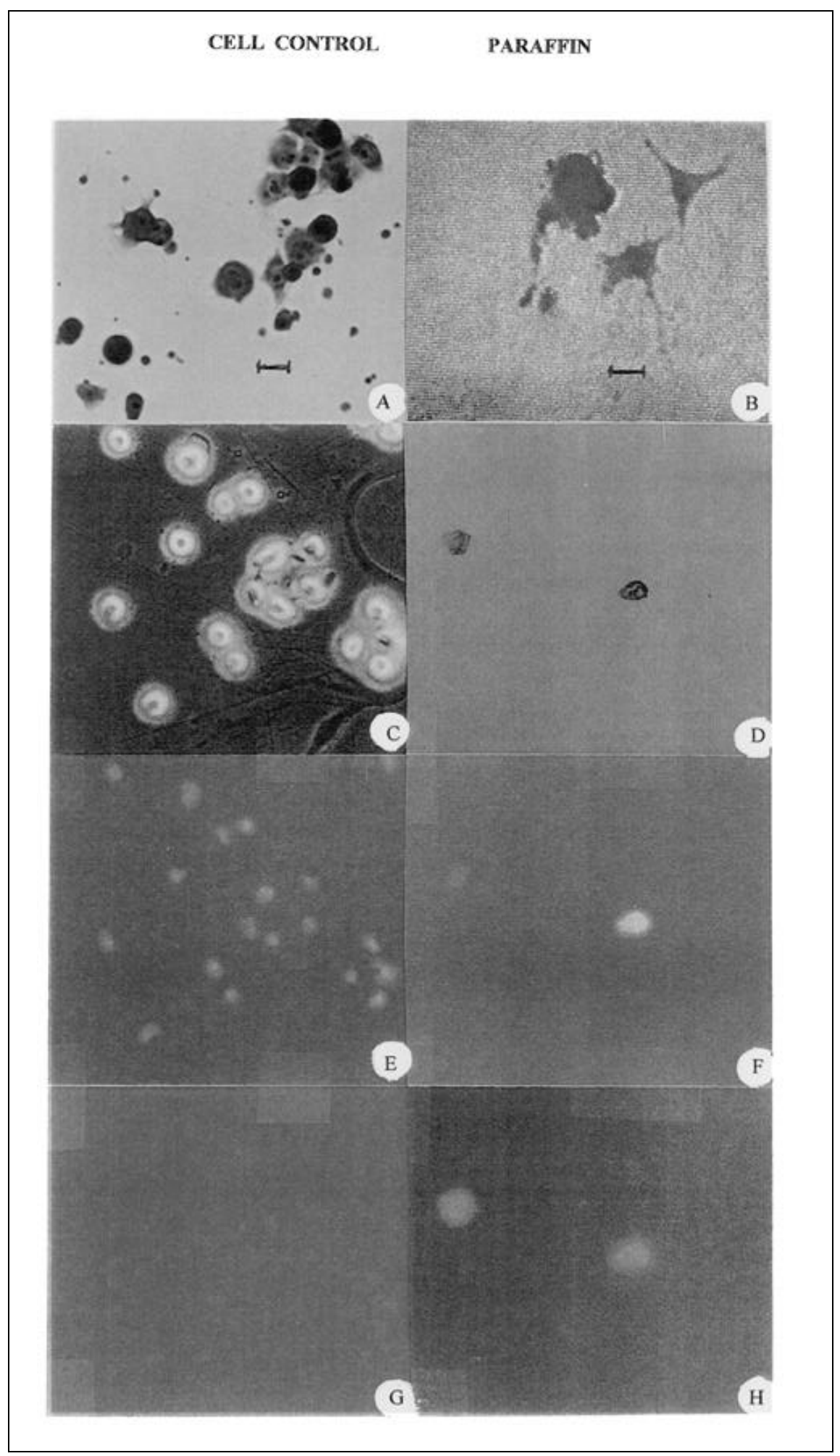

Figure 1. Visible, phase-contrast and UV results of phase 1 and 2 of experiments. Whereas photos on the left are RD cell controls, to the right, RD cells are grown on paraffin. Panel A, a visible light micrograph $(2 \mathrm{~h})$, shows normal refractile cells with visible nucleoli. Panel B (24 h) shows attached cells that are non-refractile, shrunken with dense nuclei and no visible nucleoli. Panel C (phase contrast) shows normal refractile cells, which do not take up Trypan Blue, whereas Panel D (phase contrast) shows dark non-refractile cells that stain blue. Panel E shows the uptake of the blue dye $\mathrm{HO} 342$, whereas F does not. Panel G shows no staining of cells with the red dye PI, whereas H does. Note: C, E, G and D, F, H are matching photos of the same cells in the same visual field. All photos are $400 \times$; scale bar $=25 \mu \mathrm{m}$. 
were trypsinized with $0.25 \%$ trypsin (Sigma, St. Louis, MO, USA), and the plates were seeded with 400000 cells/well in $4 \mathrm{~mL}$ of media. This was a sufficient number of cells to ensure cell-to-cell contact and to establish a confluent monolayer in several days. The cells were incubated at $37^{\circ} \mathrm{C}$ in a humidified $6 \% \mathrm{CO}_{2}$ incubator for 2 or $24 \mathrm{~h}$ or both, depending on the experiment being conducted. Cells and media were obtained from BioWhittaker (Walkersville, MD, USA). Second, histochemical studies were performed to see if PCD was the mechanism by which paraffin was killing cells. We used three viability assays concurrently to ensure a higher degree of certainty. We chose to assess both integrity of the plasma membrane using exclusion of Trypan Blue and propidium iodide [PI] and DNA degradation (failure to maintain DNA staining with the benzimidazole dye Hoechst 33342 [HO342]). PI and HO342 were both obtained from Sigma. The protocol for this was a modification of Pollack and Ciancio's method (8). Subsequently, the wells were washed with phosphate-buffered saline (PBS), fixed with absolute methanol and washed with distilled water. A separate series of plates (plain and paraffin coated) were stained with $0.25 \%$ Trypan Blue (BioWhittaker). Live cells stain blue with HO342, which is a live stain fluorochrome that binds to the minor groove of DNA, particularly to poly(dA-T) sequences (4). Because live cells have an intact cell membrane, they do not take up PI. As cells start to undergo apoptosis, the DNA breaks down, and cells cannot retain the blue HO324, which leaks out of the damaged cell membrane. Concurrently, PI enters the cell staining it red. Live cells do not take up Trypan Blue, whereas dead cells pick up the blue stain. In general, live cells scatter light and are refractile, whereas dead cells appear darker. This is particularly true under phase-contrast microscopy. UV/ phase-contrast microscopy was performed at MVI (Avon, MA, USA) using a TE-300 Inverted UV/Phase Contrast Microscope (Nikon, Melville, NY, USA). In addition, a terminal deoxynu-

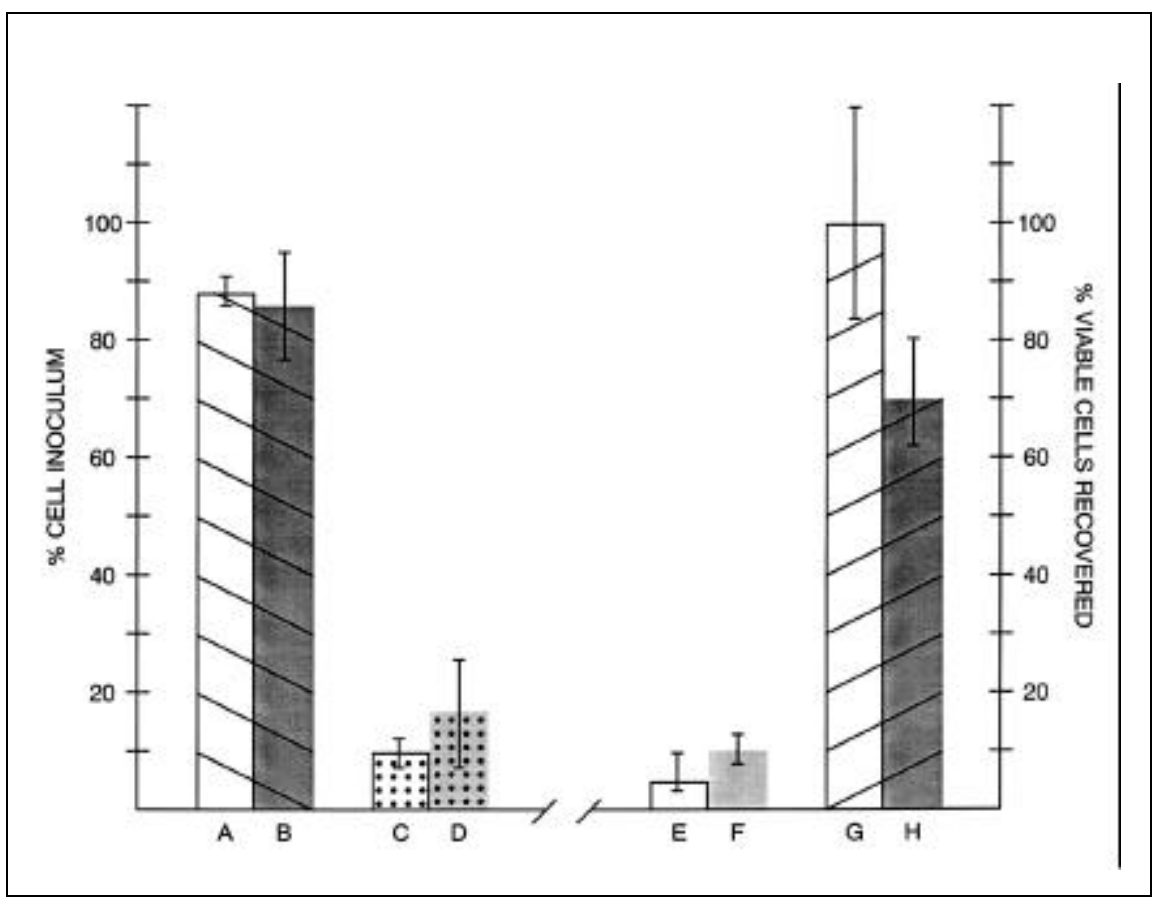

Figure 2. Cell density, viability and recovery results of phase 3 of experiments. To the left of the broken $\mathrm{X}$-axis is the cell density data; to the right is the viability and recovery data. Bar A is the percent of cell control on the surface of well after $24 \mathrm{~h}$. B is the same on paraffin-coated wells. C and D represent the percent of cells (live and dead) found in the supernatant of wells A and B, respectively. E and F are the percent of viable cells found in the supernatant of $\mathrm{C}$ and $\mathrm{D}$ transferred separately to new plain polystyrene wells. $\mathrm{G}$ and $\mathrm{H}$ represent the percent increase of $\mathrm{E}$ and $\mathrm{F}$ after $48 \mathrm{~h}$ additional incubation. 
cleotidyl-transferase (TdT)-mediated fluorescein-conjugated (dUTP) nickend labeling (TUNEL) experiment was performed using the ApopTag ${ }^{\mathrm{TM}} \mathrm{Kit}$ No. S7160 (Oncor, Gaithersburg, MD, USA) to determine nuclear fragmentation. Third, a separate cell density, viability and recovery study was conducted to determine whether or not the inoculated cells remained attached on the paraffin surface. In two parallel experiments using RD and HTB-22 cells, an inoculum of one million cells was placed on both control and paraffincoated wells and incubated for $24 \mathrm{~h}$. After the entire supernatant was collected, the cells were counted, and viability was determined by Trypan Blue exclusion. The cells were then transferred to new plain wells and allowed to grow for an additional $48 \mathrm{~h}$, at which point, the cells were fixed with absolute methanol, stained with Giemsa and counted. Fourth, to determine whether or not paraffin was intrinsically toxic, we grew cells on Falcon Model 3091 polyethylene teraphthalate filter inserts (pore size $3.0 \mu \mathrm{m}$; Becton Dickinson Labware) in close proximity, but not in contact with, the paraffin on the bottom of the well.

The results for phases 1 and 2 are shown in Figure 1. Light micrographs $A$ and $B$ show the morphological differences between cell controls and paraffin-coated wells. Panel A (2-h incubation) shows normal cells, which are refractile and in which nucleoli can be seen. Panel B (24-h incubation) shows few cells and cell debris. The cells are shrunken, with dense nuclei, and no nucleoli are visible. In addition, photos of cells on paraffin at $2 \mathrm{~h}$ incubation (not shown here) are similar to Panel B, indicating that this process occurs early on as cell death does in apoptosis. The remaining photos of Figure 1 are histochemical data (2-h incubation). Cell controls $(i)$ are refractile, (ii) exclude Trypan Blue (Panel C, phase contrast), (iii) take up HO324 (Panel E) and (iv) exclude PI (Panel G). However, cells on paraffin $(i)$ are not refractile, (ii) take up Trypan Blue (Panel D, phase contrast), (iii) exclude HO324 (Panel F) and (iv) take up PI (Panel H). In addition, TUNEL results (data not presented here) indicate DNA fragmentation only on paraffin-coated wells. In Figure 2, the third phase of the experiment, the combined data, on average, shows that after $24 \mathrm{~h}$ of incubation, only $14 \%$ of the initial inoculum could be recovered from the supernatant. Only $8 \%$ of the recovered cells were viable. These were replated for two days and showed a $70 \%-100 \%$ increase in number. Therefore, during the initial $24-\mathrm{h}$ period, $86 \%$ of the cells were attached or in contact with the paraffin surface. In phase four of the experiment, cells grown on Falcon Model 3091 filter inserts supported and grew normal cells. This precludes any inherent toxicity of paraffin working at a distance.

We have shown that adherent cells placed in contact with paraffin shrink and lose refractility. Their nucleoli become dense and their cell organelles disappear. Eventually, the cells lyse, leaving only debris. These events can be seen at both 2 and $24 \mathrm{~h}$. These visual and temporal clues, together with the histochemical data (uptake of PI and
Trypan Blue and exclusion of HO324) and TUNEL data positive for nuclear fragmentation provide evidence of an apoptotic process $(4,7,8)$. This process could be anoikis, an anchorage-dependent apoptosis that results when cells are denied their normal substratum (9). Cell death comes after separation from the surface. Because of the smooth and hydrophobic nature of paraffin, we believe that cells attaching to paraffin cannot flatten and spread-a necessary condition for cells to grow as D.E. Ingber points out in his tensegrity model (6). A change in the cytoarchitecture of the cell could induce either apoptosis or anoikis. Our results (Figure 2) show only $6 \%$ dead cells in the supernatant after $24 \mathrm{~h}$. This is evidence of attachment before death or apoptosis rather than anoikis. We have additional preliminary data that other surfaces, such as agar and polystyrene bacteriological plates, that were not treated for tissue culture, yield the same results. This would be of interest for future studies. However, at this point it is not our task to prove definitively the operative mechanism, but rather to present a new method of inducing cell death. The use of paraffin and related substances provides a quick, inexpensive, nontoxic and local method for investigating PCD and for killing cells in situ.

\section{REFERENCES}

1.Allan, D.J. and B.V. Harmon. 1986. The morphological categorisation of cell death induced by mild hyperthermia and comparison with death induced by ionizing radiation and cytotoxic drugs. Scanning Electron Microscopy 3:1121-1133.

2.Arends, M.J., R.G. Morris and A.H. Wyllie. 1990. Apoptosis: the role of endonucleases. Am. J. Pathol. 136:593-608.

3.Cryns, V.L. and J. Yuan. 1998. The cutting edge: caspases in apoptosis and disease, p. 177. In R.A. Lockshin, Z. Zakfri and J.L. Tilly (Eds.). When Cells Die: A Comprehensive Evaluation of Apoptosis and Programmed Cell Death. John Wiley \& Sons, NY.

4.Haugland, R.P. 1998. Molecular Probes Handbook of Fluorescent Probes and Research Chemicals, 6th ed., p. 151. Molecular Probes, Eugene, OR.

5.Hockenberry, D., G.T. Nunez, C. Millman, R.D. Schreiber and S.J. Korsmeyer. 1990. Bcl-2 is an inner mitochondrial membrane protein that blocks programmed cell death. Nature 348:334-346.

6.Ingber, D.E. 1993. Cellular tensegrity: defining new rules of biological design that govern 
the cytoskeleton. J. Cell Sci. 104:613-627.

7.Perry, S.W., L.G. Epstein and H.A. Gel-

bard. 1997. Simultaneous in situ detection of apoptosis and necrosis in monolayer cultures by TUNEL and trypan blue staining. BioTechniques 22:1102-1106.

8.Pollack, A. and G. Ciancio. 1990. PI exclusion coupled with HO342 staining, p. 19-24. In Z. Darzynkiewicz and H.A. Crissman (Eds.), Flow Cytometry. Academic Press, San Diego.

9.Ruoslahti, E. and J.C. Reed. 1994. Anchorage dependence, integrins and apoptosis. Cell 77:477-478.

10.Smith, C.A., G.T. Williams, R. Kingston, E.J. Jenkinson and J.J.T. Owen. 1989. Antibodies to CD3/T-cell receptor complex induce death by apoptosis in immature $\mathrm{T}$ cells in thymic cultures. Nature 337:181-184.

The authors would like to thank Mary Matson Kelly for assistance with and comments on the manuscript. Address correspondence to Dr. Thomas A. Kelly, 201 Wickham Way, \#202, Norwood, MA 02062, USA.

Received 27 October 1998; accepted 4 February 1999.
Thomas A. Kelly, Mitchell S. Felder and Robert A. Ollar Infectech

Sharon, PA, USA

\section{"Microprep" Method for Rapidly Isolating Plasmid DNAs for Restriction Enzyme Analysis}

\section{BioTechniques 26:868-870 (May 1999)}

Screening of plasmid cDNA libraries $(1,2)$ can result in hundreds of positive recombinant clones containing varying lengths of $5^{\prime}$ sequence information. Determining which plasmid contains the longest cDNA insert from a large population of heterogenous plasmids can be accomplished by either polymerase chain reaction (PCR) amplification of the $5^{\prime}$ end using forward vector and backward gene-specific primers, or by sequence or restriction enzyme analysis of purified miniprep plasmid DNA. Unfortunately, PCR amplification of the $5^{\prime}$ end frequently fails. For large numbers of recombinant clones, commercial miniprep kits can be prohibitively expensive, and most 'rapid' miniprep procedures involve multiple steps and reagents that prove to be tedious and time-consuming. To overcome these obstacles, we have developed a rapid and cost-effective microprep procedure using microliters, rather than milliliters, of bacterial culture for rapidly isolating plasmid DNA that is suitable for restriction enzyme analysis.

The procedure is described in Table 1. Figure 1 shows an analytical gel with typical results.

Use of the procedure to prepare DNA for screening saves use of the larger miniprep or maxiprep procedure for preparing only the desired clones. Agarose gel analysis of restriction enzyme digested microprep DNA results in ethidium bromide (EtdBr)-stained bands that are easily resolved from high-molecular-weight bacterial, chro- mosomal DNA and small-molecularweight bacterial RNA (Figure 1). SalI and NotI restriction endonuclease digested microprep DNA prepared from ${ }_{\text {DH10B }}{ }^{\mathrm{TM}}$ cells (Life Technologies, Gaithersburg, MD, USA) equally well in both $1 \times$ Buffer $\mathrm{H}$ (Boehringer Mannheim, Indianapolis, IN, USA) and $2 \times$ Universal Buffer (Stratagene, La Jolla, CA, USA). However, we have not checked the impact of using other bacteria, restriction enzymes or buffers on microprep DNA quality. It would be prudent for an investigator to run a pilot microprep analysis with their particular bacterial strain(s) and restriction digestion conditions before scaling-up the microprep procedure.

In summary, we have developed a streamlined, microscale approach for isolating plasmid DNA from overnight

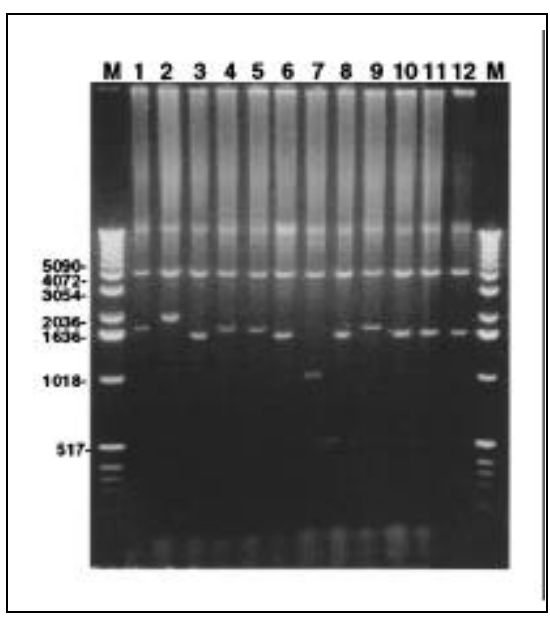

Figure 1. Restriction enzyme analysis of multiple heterogenous plasmid cDNAs isolated by the microprep method. Plasmids containing rabbit Kv3.3 cDNA cloned into the SalI/NotI restriction sites of pSPORT1 vector (Life Technologies) were enriched ( $>95 \%$ positive) from a corneal epithelium plasmid cDNA library (3) by the magnetic bead-capture approach (2). Plasmid DNAs were isolated from DH10B cells by the microprep method as described in the text. Restriction enzyme digestion was performed in $20 \mu \mathrm{L}$ vols consisting of $17.8 \mu \mathrm{L}$ of supernatant, $2 \mu \mathrm{L} 10 \times$ Buffer $\mathrm{H}$ (Boehringer Mannheim), $0.1 \mu \mathrm{L}$ SalI and 0.1 $\mu \mathrm{L}$ NotI $(10 \mathrm{U}$ per $\mu \mathrm{L}$ stocks; both from New England Biolabs, Beverly, MA, USA) followed by incubation at $37^{\circ} \mathrm{C}$ for $1 \mathrm{~h}$. A $4 \mu \mathrm{L}$ vol of $6 \times$ Ficoll ${ }^{\circledR}$ gel loading buffer (Type II) (1) was added to each tube and $6 \mu \mathrm{L}$ of the resulting mixture were electrophoresed using a $1 \%$ agarose $/ 0.5 \times$ TBE gel with $40 \mu \mathrm{g} / \mathrm{L}$ EtdBr. Lane M, 1-kb DNA ladder (Life Technologies); lanes 1-12, plasmid cDNAs. Marker band sizes are indicated in bp. SalI/NotI double-digested of pSPORT1 is $4.1 \mathrm{~kb}$, and insert sizes range from 1-2 kb. 